\title{
WHITEHEAD AND RUSSELL'S THEORY OF DEDUCTION AS A NON-MATHEMATICAL SCIENCE
}

\author{
BY E. J. NELSON
}

1. Introduction. In his paper in the June, 1931, issue of this Bulletin, B. A. Bernstein attempted to transform the theory of logic in Whitehead and Russell's Principia Mathematica into a mathematical science. $\dagger$ In this paper, I wish to discuss the general question whether or not this theory can be stated as a mathematical science. I shall use Bernstein's exposition as a guide in the discussion, because it not only states in admirable form and simplicity the nature of a mathematical science but also either brings up explicitly or at least suggests each of the issues involved.

2. Nature of a Mathematical Science. In the first place I shall summarize his account of the nature of a mathematical science, by which he says he means a pure deductive theory.

(1) It is "a body of propositions consisting of postulates and theorems."

(2) These propositions "give information about a certain class of elements and about certain operations or relations among the elements."

(3) "The classes, operations, and relations constitute the ideas of the science," some of which are taken as primitive, and the others of which are defined in terms of the primitive ones.

(4) "Every proposition must contain, besides the ideas belonging to the science, also ideas that are outside the science." This is necessary in order that the propositions may give information about the ideas within the science. The ideas outside the science are those of "general language".

(5) "Since the theorems are derived from the postulates, the science must use, beside the propositions belonging to it, also

$\dagger$ Whitehead and Russell's theory of deduction as a mathematical science. In vol. 35, No. 1, pp. 301-303, of the Transactions of this Society, E. V. Huntington made a similar attempt to mathematicize the logic of the Principia. His result is subject to the same general criticisms as I present in this paper against Bernstein's transformation. 
propositions which are outside it"; namely, "the principles of logic which give the theorems as conclusions from the postulates as premises."

(6) The science is abstract, in the sense that our primitive ideas derive all their specific properties from the postulates; and hence the system is a "logical skeleton" for which several interpretations are possible.

3. Criticism of the Principia. Among the shortcomings of the Principia as a mathematical science our author finds the following:

A. Assertion $(t)$ "does not stand for any class or operation or relation." And further "nothing is said about it, no condition is imposed on it, by the primitive propositions."

B. Proposition *1.1 ("anything implied by a true elementary proposition is true") fails because, having no symbols, it has no ideas belonging to the theory and hence says nothing about the ideas of the theory. That is, it imposes no conditions upon any of the primides. $\dagger$

C. $* 1.2$ to $* 1.6$ fail too, for the same reason, though in an opposite manner; namely, because they contain nothing but ideas within the theory and hence say nothing about these ideas. In other words, these postulates impose no conditions upon the primides, for without the use of other notions the primides can not impose limits upon themselves. Thus we find that the only postulates that conform to the requirements for postulates in a mathematical science are $* 1.7$ and $* 1.71$.

Now I shall indicate and discuss Bernstein's corrections which he believes will give to the theory of the Principia the nature of a mathematical science.

A. Assertion. If he is right in holding that $\vdash$ does not stand for any class, operation, or relation, and that the postulates impose no conditions upon it, then he cannot at the same time be right in holding that only $* 1.7$ and $* 1.71$ contain ideas outside the system, for by his own criteria, $\vdash$ must be outside the system

† Just as we have the word "postulate" for " primitive proposition," I suggest coining the word "primide" (adj., primidic) to mean what is meant by "primitive idea." Besides serving the interests of brevity and having an adjectival form, this word has the derivational suggestiveness of the component notions of the idea for which it stands. 
in spite of Whitehead and Russell's explicit assertion that it is one of their primides. Bernstein certainly is right when he says that the properties of $\vdash$ are not given by the postulates in the manner usual in mathematics. Hence the upshot of this seems to be (1) that the authors of the Principia used a symbol for a notion which, by the customs in mathematical circles, should have been expressed by an ordinary word or sign; and (2) that, in spite of their statements to the contrary, assertion is not a primide in their system.

Bernstein's correction on this point is to write " $p=1$ " instead of ".$+ p$," where " $p=1$ " means " $p$ is true." The equality sign stands for truth-value equivalence; thus, $p=q$ means that $p$ and $q$ are both true or both false. The sign " 1 " stands for a certain, though undesignated, member of the $K$-class. Accordingly $p$ and 1 are ideas within the system, whereas $=$ is not. This last then provides an idea external to the system, which we may employ in our propositions and in terms of which we can say something about our primides.

Employing this notion of equivalence, and Boolean notation, Bernstein translates the primitive propositions into mathematical form. For purposes of reference in the sequel, I interpose here enough of his postulates to indicate his procedure.

"Consider an undefined class $K$ of elements $p, q, r, \cdots$. Let $p^{\prime}$ be the result of an undefined unary operation on a $K$-element $p$, and $p+q$ the result of an undefined binary operation on the $K$-elements $p, q$. The theory of deduction is the $\operatorname{system}\left(K,{ }^{\prime},+\right)$ satisfying the postulates 1.1-1.71 below.

1.1. There exists a $K$-element 1 such that from $p=1$ and $p^{\prime}+q=1$ follows $q=1$.

1.2. $(p+p)^{\prime}+p=1$.

1.6. $\left(q^{\prime}+r\right)^{\prime}+\left[(p+q)^{\prime}+(p+r)\right]=1$.

1.7. If $p$ is a $K$-element, $p^{\prime}$ is a $K$-element.

1.71. If $p$ and $q$ are $K$-elements, $p+q$ is a $K$-element."

B. The Principle of Inference. We now return to Bernstein's criticisms. Postulate $* 1.1$ presents, I think, the critical point of the whole argument. Whitehead and Russell say it can not be expressed symbolically. Bernstein undertakes to do so, as indicated above. Has he succeeded? 
(a) In the first place let us notice that he has employed the notion represented by "follows" (i.e., "therefore," not "implies"), which is obviously outside his system. The legitimacy of this in a theory of deductive logic seems questionable, for is not the notion of "follows" essential to a deductive system of logic? Without it how can we ever derive a theorem from a postulate? A somewhat similar difficulty arises in regard to his leaving equivalence outside the system, since this notion of same truth-value of propositions is fundamental in the extensional logic of the Principia (and can scarcely be said to be an idea of general language). Hence, to exclude it from the system of deduction is simply to omit part of that system. And again a case of this kind of difficulty arises in his employment of the notion of "If . . then" in 1.7 and 1.71: he keeps it outside his system, but assuredly it represents a fundamental logical idea.

(b) In the second place, if we suppose with Bernstein that his 1.1 corresponds to the Principia's*1.1, then how can theorems ever be derived from the postulates, without still some other postulate $(\alpha)$ in virtue of which the theorem follows from 1.1 and (say) 1.2 and 1.6? But still another principle $(\beta)$ is now needed to justify the inference of the given theorem from $(\alpha), 1.1,1.2$, and 1.6; and so on endlessly. The problem here is the one made famous by Lewis Carroll's account of "What the Tortoise said to Achilles," $\dagger$ and solved by Mr. Russell's principle of inference. $\ddagger$ The point is that the principle of inference cannot be used as a premise, or as an ordinary postulate, but must be an instrumentality or justification, apart from the premises or postulates, by authority of which the implicate of the premises is made a conclusion; that is, is asserted independently of the premises or postulates. The conclusion is not inferred from a set of premises among which is the principle of inference, but from the premises by virtue of the principle of inference.

The lesson Carroll and Russell have taught us is-applied to any deductive system - that our primitive propositions must be of two kinds: (1) Those that function like ordinary mathematical postulates; that is, that impose conditions upon the primides; and (2) those in virtue of which we drop premises and

$\dagger$ Mind, n.s., vol. 4 (1895), p. 278.

† See Russell, The Principles of Mathematics, vol. I, \$38. 
assert categorically what is implied by them. The mathematical sciences formulate only those of the first kind, but employ also those of the second, which, borrowed from logic, they take to be outside their systems. But since those of the second kind are principles of logic (e.g., the principle of inference) a formulation of logic as a deductive system must not leave them out.

As a matter of fact, when we deal with a system of logic, the situation is even more involved than this, for in such a systemas contrasted with a system of mathematics - the logical principles of type (1), though not of type (2), have a two-fold office; namely, as principles of deduction and as premises. For example, if the principle of the syllogism be chosen as a postulate, then in deriving theorems we might in the same step employ it as a premise and as a principle of deduction used upon itself as premise. For instance, let two of our postulates be (1) $p \supset q \cdot q \supset r \cdot \supset \cdot p \supset r$, and (2) $p \supset r \cdot \supset \cdot \sim r \supset \sim p$. Hence, by authority of (1) used as a principle of deduction, we infer from (1) and (2), both used as premises, the theorem $p \supset q \cdot q \supset r \cdot \supset$. $\sim r \supset \sim p$, employing in addition a principle of inference. From the point of view of deducing theorems, the postulates in $* 1$ of the Principia may be divided into three groups: (a) $* 1.1$ and *1.11, which are Principles of Inference; (b) *1.2-*1.6, which I call Principles of Deduction; and (c) $* 1.7-* 1.72$, which are not used in proofs in the ordinary sense, but function rather as definitions or characterizations of certain kinds of elements of the $K$-class, determined by operations or relations upon other elements of this class. See *3.03.

Whitehead and Russell's language in distinguishing between the two types of principle was unfortunate. In one sense, if a principle can be stated at all, it can be formulated in symbols. But they likely had in mind implicitly the truth that their principle of inference contains at least one idea which has no place in any of the other primitive propositions and which is not a primide in the precise sense the others are; namely, the notion of "inference" or "therefore." Had they simply pointed out that their primitive propositions are of two distinct types, confusion might have been avoided.

In discussing the Principia in the Nov.-Dec., 1926, issue of this Bulletin (pp. 711-713), our author stated that "The logic 
of propositions is simply† a two-element logic of classes, . . . " If we be right in holding with Russell and Whitehead that there are two kinds of principle, then this quoted statement must be in error, for the algebra of classes, or of the truth-values 0,1 , does not contain a principle corresponding to the Principle of Inference; hence any system having a Principle of Inference within it cannot be simply a logic of classes.

4. Conclusion: Logic cannot be Mathematicized. By the foregoing I think we are driven to conclude that Bernstein has not mathematicized the logic of the Principia. And if to be a mathematical science means to have the characteristics set forth by him, then I seriously doubt that logic can be mathematicized. In expounding the nature of a mathematical science, he explicitly asserts that the principles of logic must be outside the system in order that theorems may be derived from postulates. But how can we leave the principles of logic outside the system of logic? If his characterization of a mathematical science be correct, then it seems that the theory of deductive logic cannot be mathematicized, for it cannot be this theory if it omit part of itself, and, according to our author's criteria, it must omit part of itself if it is to be a mathematical science. Hence, it is Bernstein's own requirements Nos. 4 and 5 that preclude the very possibility of his achieving his purpose to mathematicize logic.

If, however, we be permitted to have two types of postulate, and further if we may take as primidic all technical logical notions employed but not defined in terms of others, then we shall have some sort of deductive system and also a precise formulation of logic. Though we may not have mathematicized it, may we not say we have logisticized it?

5. An Objection, and Reply. I wish to consider here a possible answer to my objections; namely, that I have failed to distinguish between the symbols of the logic and the notions outside the logic. Thus, it may be said that " $\supset$ " is a symbol belonging to the logic, but "implies" is outside the logic, as in " $p \supset q$ and $q \supset r$ ) implies $(p \supset r)$." Similarly, it may be objected that I have confused the logical principles (postulates and theorems) symbolized and in the system of logic with these principles unsym-

$\dagger$ Italics mine. 
bolized and outside the system, which underlie the symbolized logic. Bernstein made this point against Schröder, Whitehead, and Russell, in volume 28 of the Transactions of this Society.

My rejoinder is that it is just this underlying or "sub-logic" logicians have meant to symbolize. If Bernstein's point is well taken then there must be an infinite number of logics, for no matter what logic I formulate, he finds another underlying it. In the existence of an infinite hierarchy of logics there may be no theoretical difficulty, provided it is theoretically possible to find points of difference between them so that, if I were presented with two such logics, I should be able at least to see that they are two, let alone be able to determine which is $s u b$ and which super. But this difference has not been pointed out. When I endeavor to differentiate between the principle of the syllogism belonging to the system and the principle of the syllogism belonging to the sub-logic, I find only one principle. Reflecting upon the principle of the syllogism unformulated in symbols (that is, in the sub-logic) and upon the one formulated in the symbols of the system, I find no difference between them. On the contrary, I discover merely two statements expressing one and the same proposition. A similar situation seems true mutatis mutandis of the other laws of logic.

Furthermore, the notion symbolized by $\supset$ seems to be identical with the idea named "material implication." Hence I can hardly have confused the meaning of $\supset$ with the meaning of "materially implies," for there is only one meaning. We must take care not to confuse symbols with the symbolized. I am not unaware of Russell's doctrine of the "systematic ambiguity" of his primides; for example, of disjunction. But the logical notion I am interested in is that essence or property common to all the $o r ' s$ which justifies us in some way grouping them together and which keeps us from confusing an or of one type with (say) a not of some other logical type. If, however, it be replied that there is no such essence, and that to suppose there is is to fail to grasp what Russell talks about when he uses the term "systematic ambiguity," then this reply presupposes a nominalism of the most extreme form; it reduces all logic and mathematics to nothing more than pencil marks on paper, or sounds, which are not symbols and cannot be discriminated in any way relevant to logic or to mathematics.

Similarly, if Bernstein has in mind a series of logics such as 
would be demanded by Russell's theory of logical types; namely, such that propositions of the $n$th type would occur in a logic about propositions not higher than the type $n-1$, if this is what he means, then the logic I am interested in is the logic which he uses in talking about these different types of logic. But as he of course knows, Russell's theory of types would appear to make even the supposition of such a logic nonsense, for any proposition applicable to all types of propositions would be nonsense.* Accordingly, his implication that for every formulated logic there is a sub-logic would be such a statement, and nonsense. But since I think I am safe in saying that we both agree that he was not talking nonsense, I conclude that he admits there are propositions, and hence a logic, applicable to all types. These considerations seem to dispose of the possibility that I have misunderstood him in regard to the theory of types. $\dagger$

6. The Principle of Inference. Bernstein's treatment does, nevertheless, suggest to me a view which might contain an element of truth; namely, we might hold that the principle of inference in the Principia is a theorem or a postulate in a superlogic, in which logic, however, it does not function as a principle of inference. From this it would follow that a principle of inference used as such in any deductive system does not belong to that system as a postulate or theorem but to some other system in which it does not function as a principle of inference. Thus, the principle of inference in the Principia would be of a higher type than the premises of arguments in which it is employed. And if this be the case, my fundamental point is further substantiated; namely, that the logic of the Principia, containing such a principle, cannot be made into a mathematical sci-

* See Weiss, The theory of types, Mind, n.s., No. 147.

$\dagger$ The use of "sub-logic" in the above sense would seem psychologically justified, for we think of such a logic as more "fundamental" or basic or underlying. But from the standpoint of the theory of types it seems that the principles that justify inference in a given logic would have to be principles belonging to a logic of higher type. For example, "If $p$ and ' $p \supset q$ ' are propositions of type $n$, then $q$, which is likewise of type $n$, follows" would be such a principle, and would be of type $n+1$.

I should like to caution the reader that by the foregoing discussion I do not mean to imply that I think the Principia Mathematica presents the logic of propositions. I believe that there is an intensional logic more fundamental than extensional systems. But this in no way compromises what $I$ have said, for the questions under discussion might apply as well to the intensional system. 
ence. Accordingly, Bernstein should not have restated $* 1.1$ as 1.1 , but rather he should have excluded it from the system entirely, explaining that inferences within the system proceed in conformity with some principle outside the system, as provided by one of his own requirements. This is the best case I can make out for Bernstein's general point of view, though of course it is not compatible with his specific treatment, for he recasts the system for the purpose of showing that the principle of inference is within the system. If this suggestion be sound, then it is an explanation of my previous point that the primitive propositions of the Principia are of two kinds.

7. Independence Proof. If our principal arguments so far are sound, I think we can see now the truth of Whitehead and Russell's statement that "The recognized methods of proving independence are not applicable, without reserve, to fundamentals." $\dagger$ Since in proving independence we must employ valid forms of deduction (for example, the principle of transposition, or the law of contradiction), any independence proof of the postulates of a set composed of such logical laws would be circular. If, however, we take the Principia's set of postulates, omitting *1.1 and *1.11 since they are of a kind different from the others, then, considering this set as a system-form, that is, abstracting from the interpretation of them as principles of logic, we may perform the usual independence tests. Might it not be that this condition is what the authors of the Principia referred to by the phrase "without reserve"? Moreover, if again we exclude $* 1.1$ and *1.11, it seems that the circularity mentioned above is neither more vicious nor different in kind from that involved in any deduction of logical principles from logical principles by use of the latter logical principles as valid forms of deduction.

Accordingly I am unable to see how to avoid circularity in a deductive system of logic, though it is important to notice that the apparent viciousness of such a circle is not real, because every postulate of logic stands on its own feet; that is, it is true a priori, and is "begged" only in the sense that it is chosen as a starting point.

The University of Washington

$\dagger$ Principia Mathematica, p. 91. See Russell, The Principles of Mathematics, $\$ 17$. 